\title{
The Conscious Internet: An Empirical Study of the Transmission of Healing Energy via E-mail
}

\author{
Francesca McCartney* \\ EMU Founder, President, Energy of Medicine University, USA.
}

*Corresponding author: Francesca McCartney, EMU Founder, President of Energy Medicine University, USA.

Received Date: June 26, 2018

Published Date: August 23, 2019

\section{Abstract}

This research studies the intentional encapsulation of healing energies into an email text message and transmittal via the Internet to an intended intuitive recipient. This study framed within the field of energy medicine researches the potential impact on the learning of distance students; as well as investigates a method for a stronger link-transference of information between distance educators and students. This study tests information discovery on non-linear, non-local communication; as well as studies an implied evolution of the sense of intuition.

Distance education and intuitive communication are both non-linear, non-local systems of information exchange. Internet email can hold intentional sensorial resonance; and this type of email can be used as a provider of energy-information which can be utilized as part of an energy medicine distance education program. An intuitive person can open an Internet transmitted email and receive the emotional/cognitive content of intentionally encapsulated energy/information; and this occurs both as a non-local and non-linear experience.

The Internet is an evolving ecological system capturing and holding human thought in digital format trans versing time and space in order to deliver content. The weaving of intuitive and Internet communication is spawning a World Wide Web, small-world phenomena by shortening energetic time-space distances between people.

Within this research is a hypothesis that a student and teacher may be more strongly connected at a distance with the intentional link of energy/ intention included within the curriculum of a distance education program. This linkage information gives new significance to distance healing research within the medical, parapsychological, and noetic sciences. This connection seeks to inspire and identify fertile areas for cross-disciplinary exploration.

Keywords: Education; Energy medicine; Extra-sensory perception; Internet; Intuition; Neural networks; Psychology

\section{The World Wide Web-A Collective Consciousness}

The Internet is a network which is spawning a quantum leap in human consciousness. The blending of digital reality with human reality blurs the distinction between electric-based and biological-based systems. The World Wide Web of the Internet collapses distance, time and proximity in human communications and creates a unified state of constant connectedness. Casting an invisible net over the globe, the World Wide Web is collecting and shortening the spaces between individuals. The Erdos concept of "link-chains" in social network research which studies short distances between people is popularly called, "sixdegrees of separation." According to physics professor, Albert Laszlo-Barabasi, an Internet distance (clicks) between people is nineteen-degrees of separation; and the present social network distance is three-degrees of separation. Who is the caster of this net? Are those who are gathered into this web net conscious of the implications of a global paradigm shift in human evolution? What is occurring on an individual basis within this web net, and what is being born as a whole entity?

\section{Statement of purpose}

This project explores how energy medicine and complimentary inter-disciplinary fields can be woven together in order to discover and cross-reference the effect of Internet communication, intuition, and intentionality on the human species. The focus of this research is limited to one area of experimental investigation - that of intuition-based communication via the Internet. Millions of people are sending billions of email messages via the Internet in this new world communication link which utilizes (or is using) the Internet as a host medium for non-local conversation. This form of Internetbased communication is modeling the natural human sense of intuition-based communication. Using the Internet in this way-- as 
a tangible, mental construct-- allows human psychology an "object" to grasp as a bridge to understanding the already present state of non-local, no-time, no-space, intuition-based communication.

\section{Hypothesis}

Healing energy can be encapsulated into an email text message and transmitted via the internet to an intended recipient: This hypothesis developed from this researcher's observations during the year 2000 of a five-month healing energy email Internet exchange which occurred organically between two Intuition Medicine ${ }^{\circledR}$ practitioners. From a broad perspective, this research will investigate the efficacy of non-local, no-time, no-space distance education programs that utilize intentionality and the Internet. It also identifies fertile ground in the fields of Internetbased distance education and energy-based medicine that is worthy of future exploration.

\section{The Inspiration}

The idea for this research came from an experience I had as a teacher of professional intuitives at the Academy of Intuitive Studies and Intuition Medicine® located in Northern California. I was working with two of my Master of Intuition Medicine (MIM) program students one was an alumna of the 1993 MIM program and a professor in the computer arts college of a German university. The other was an owner of a computer consulting company, and a student in the 2000 MIM program. Part of the MIM program provides each student with an alumnus mentor who assists in the course- work, and also gives weekly energy-systems healings. The student had many areas of intuitive interest that matched well with the alumna's expertise, given that they both spent a good deal of time at their computers, and on the Internet, I decided to have them connect at a distance via email as student and mentor. I assumed that they would communicate via some live chat system, forgetting that the time difference between Germany and the USA would not allow for this kind of communication. They began, quite organically, to communicate to each other via text email as though they were speaking to each other in the present moment.

The student would ask for an energy healing with specific requests regarding energy-systems, and then send the email to the mentor. The mentor would receive the message -- usually eight hours later and respond by sending both a reply email text message, and within the text "encapsulate" an energy healing and then send to the student. They were amused to discover that the sending and receiving of energy-systems healings was operating as intended- without the condition of physical proximity, and in disregard of the time that the email (which was encapsulated with the healing energy/information) was sent. When the student would read the text message with the information describing the healing action that the mentor had facilitated, she became aware that she began to feel the changes while she read the message, or realized that she had already received the pertinent energy/information previous to reading the actual email message. Communication and exchange of information and energy was occurring as a non- local, no-time and no-space occurrence, and being transmitted via intentionally encapsulated text email messages, consistently with the same reception results for five months. I theorize this as a force of "space- conditioning" existing within the Internet. I observe this as a test of "vital-reality" existing within the World Wide Web.

\section{Research Overview}

This research was a randomized, single-blind study. There were four formal treatments with 88 test subjects. The procedure had a professional intuitive encapsulate a healing energy into a CD-ROM which held a text email message. A computer program randomly transmitted the encapsulated energy email to the test subjects. Four different email treatments were created; three of them encapsulated with three different healing energies (one energy per email), and the fourth email had no encapsulation of healing energy. The email sending sequence was spaced one week apart. Each test subject received one of the three sample energies and one "no energy" email. The sending sequence of the test subject emails and the order of the four energy/no energy emails was randomized by a computer program.

\section{Methods}

Professionally trained intuitives were used as the test subject "measurement instruments" for this research. One professional intuitive (PI), who is the graduate program instructor at the Academy, intention-imprinted a healing energy onto a CD-ROM. A computer program randomly transmitted the intention-imprinted email to the test subjects. The PI performed the procedure at the Institute of Noetic Sciences laboratory isolated within a double steel-walled, electromagnetically and acoustically shielded chamber 1 in order to minimize ambient resonance, kinetic energy, and other distractions. This chamber is most often used for electromagnetic field testing and for psi experiments.

After entering the chamber, the PI spent one to two hours in meditation; during that time he separately held each of the three CD-ROMs in his hands and intentionally directed a specific healing energy through his hands directly onto the CD-ROM. After intention-imprinting each CD-ROM with a healing energy the PI placed it in the computer which sent it to the target group using a random email sending program. This process was repeated each week for four consecutive weeks.

The email sending sequence was spaced one week apart and on different days of the week. Each test subject received one of the three sample energies and one "no energy" email. The sending sequencing of the subjects' emails and the order of the four emails was randomized using a simple random number table.

The subjects were randomized in groups of four, and each week each group was re-randomized. The PI did not know who was receiving the emails, other than they were MIM alumni. The CDROMs were handled only by the professional intuitive; they were stored in the chamber, triple wrapped in aluminum foil, stored in separate tin cans which were placed in a covered cardboard box. 


\section{Sample population}

The scope of the study was limited to the specific healing energies that are taught within the Master of Intuition Medicine program at the Academy of Intuitive Studies and Intuition Medicine. Thus, the target population includes the following seven types of healing energies. Intuitives are taught a specific protocol for accessing, amplifying, directing, and utilizing these seven energies for purposes of:
- healing body/mind/spirit of self and others

- $\quad$ sourcing information (internally and externally)

- creating energetically grounded spaces (internally and externally)

- $\quad$ evolving their mind/body/spirit. In this study, a sample of three of the healing energies were used to test the research hypothesis

\section{Report of research results}

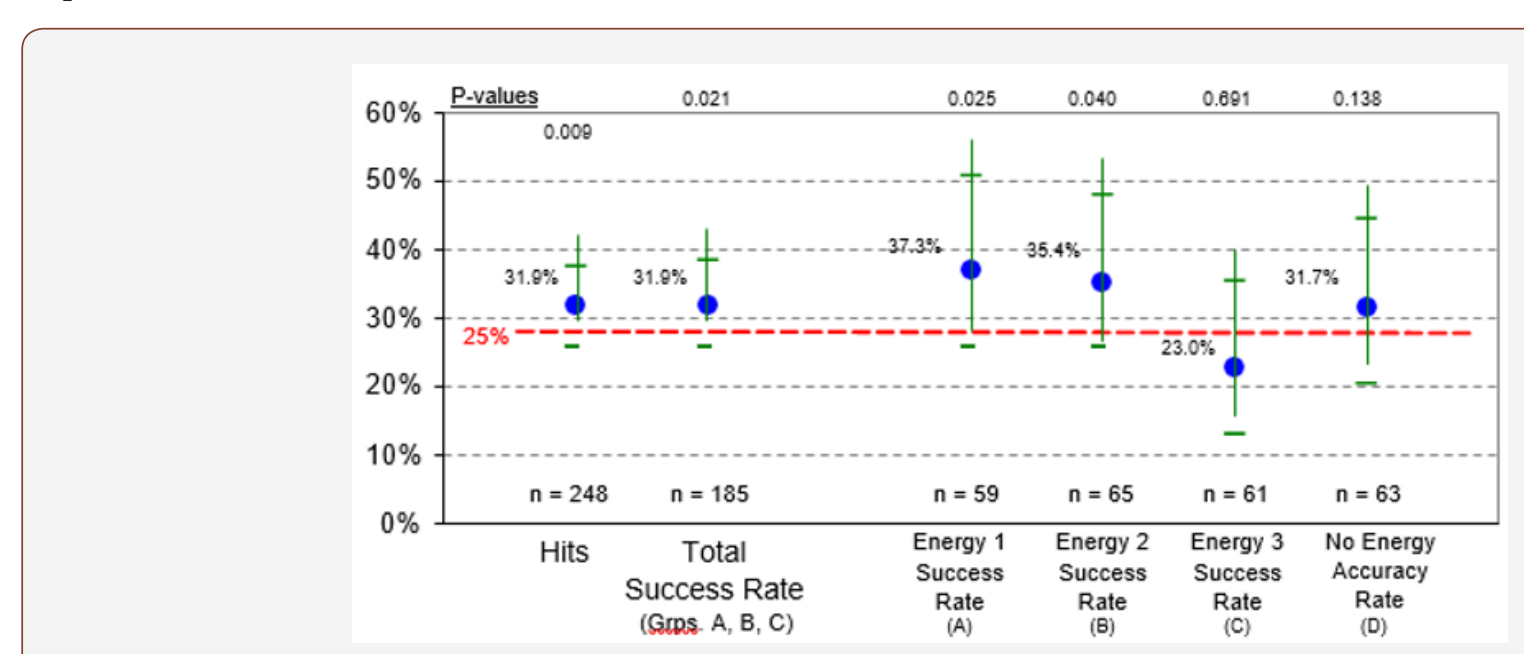

Figure 1: Display of Hits \%, Total Success $\%$ and Treatment GROUP Success \%, Estimates with $95 \%$ Confidence Intervals.

A total of 248 responses were received during all four weeks of the study. Shown in the figure below is a plot of the Hits percentage, the Total Success percentage, and the respective success percentage for each of the four treatment groups. For purposes of this study, "success" is defined as both the correct detection and identification of the encapsulated healing energy. Thus, the term "success" is strictly associated with the three treatment groups (A, B and C) that included an encapsulated healing energy. The "Total Success Rate" is calculated using the responses only from treatment groups A, B and C (total of 185). The "No Energy" treatment group (Group D) was completely void of any healing energy. Since there was no healing energy present to "identify" in this group, the percentage of correct responses is labeled "Accuracy Rate" to distinguish it from "success rate." The "No Energy" treatment group was included in the study largely to assess if the test subjects could detect both the presence and the absence of healing energy with similar accuracy. The term "Hits" is a commonly used term in ESP studies and is simply defined as the percentage of ALL total correct responses. Therefore, "Hits" does include all four treatment groups (A, B, C and D) and calculated using all 248 received responses. Relative to the $25 \%$ chance of success, the Total Success Rate percentage estimate of $31.9 \%$ is significant with a P-value of 0.021 , $(n=185)$ (Figure 1).

\section{Report of intuitive skills tested}

Of the 248 total responses received, 190 reported which intuitive skills were used. In $64 \%$ of those responses (121 out of 190) the test subjects reported using multiple (two or more) intuitive skills to detect and identify the healing energy. Only one skill was reported being used in the remaining 36\% (69 out of $190)$ of the responses. Only nine test subjects $(10 \%$ of the 88 total participants) reported using the exact same skill or combination of skills during all weeks of the study in which they participated. Most test subjects used different skills or combinations of skills each week, although the differences were usually slight. Two of the listed skills, Clairaudience and Telepathy, were never used singly by any of the test subjects. If these two skills were used, it was always in combination with other skills. The other five skills were used both singly and in combination with other skills. Following in the figure below is a bar chart of the skills and combination of skills reported used by the test subjects.

\section{Intuitive skills defined}

The Intuitive test subjects were asked to detect the presence or absence of a healing energy using their specifically developed intuitive skills. The subjects were given this list of seven intuitive skills to choose from which are specific to the method which is taught in the Intuition Medicine system.

\section{- $\quad$ Clairvoyance: Chakra-Clear Seeing}

Clairvoyance is a "visual" experience or awareness of subtle energy. The usual visuals that are perceived are colors, symbols, patterns, or images.

- $\quad$ Clairaudience: Chakra-Clear Hearing 
Clairaudience is a literal hearing of words, sounds, songs, or tones. This skill is an outer audio sound vs. inner mental thought. This intuition is also described as a human sonar sense.

- Clairsentience: Chakra- Clear Feeling

Clairsentience is an acutely developed sense of empathy. This skill utilizes the nervous system to feel energy. Clairsentience is also referred to as emotional body-radar.

- Telepathy: Telepathic Chakras-Mental Communication

Telepathic ability is communication without verbal/auditory speaking. This skill has a two-way path of sending and receiving.
- Precognition: Broad Band Intuition-Future Perception Precognition is a future time perceptual skill.

Precognitive information can be perceived by means of knowing, clairaudience, clairvoyance, telepathy, dreams, visions and clairsentience.

- Psychometry: Hand Chakras-Dowsing Perception

Psychometry is a feeling perception located specifically in the hands. Psychometry is similar to dowsing; the difference is that hand-psychometry is used without any attending objects- i.e. a water witching stick or pendulum.

- Knowing: Chakra-Abstract Perception

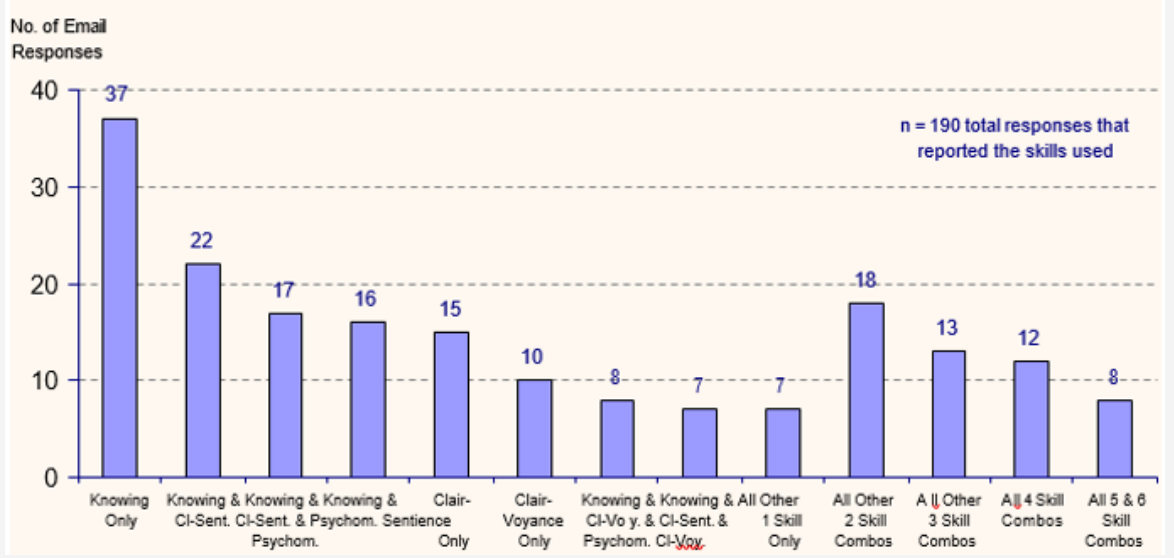

Figure 2: Bar Chart of the Frequency of Reported Skills Used to Detect and Identify Encapsulated Energy.

Intuitive Knowing presents answers and information often in no logical manner. Knowing may be experienced as a certain feeling, awareness or trust-based perception (Figure 2).

\section{Digital collective unconscious}

Millions of people are sending billions of email messages via the Internet in this new world communication link which utilizes (or is using) the Internet as a host medium for non-local conversation. This form of Internet-based communication is modeling the natural human sense of intuition-based communication. Using the Internet in this way-- as a tangible, mental construct-- allows human psychology an "object" to grasp as a bridge to understanding the already present state of non-local, no-time, no-space, intuitionbased communication. Psychologist Carl Jung's work- the collective unconscious is another concept of an intuitive storage matrix of human information. Jung's realm of the collective unconscious holds mental forms of psychological structure which he calls archetypes: the Self, the Anima/Animus, the Shadow are a few. Lesser known is Jung's view of small integer numbers (1, 2, and 3) as archetypes. Numbers, he suggested, are the basic abstract structures from which all archetypes are built. Jung may have been a futurist espousing a psychological-digital-reality of cyberspace.

\section{Global mind and global spirit: is gaia evolving?}

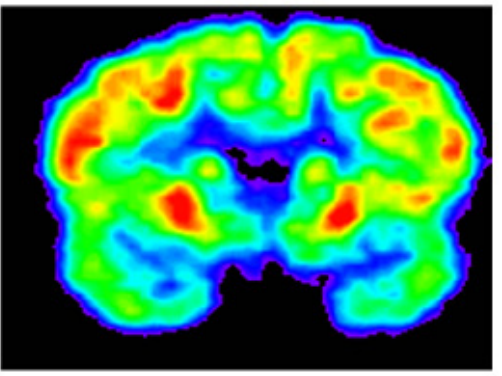

a. PET scan image of the human brain

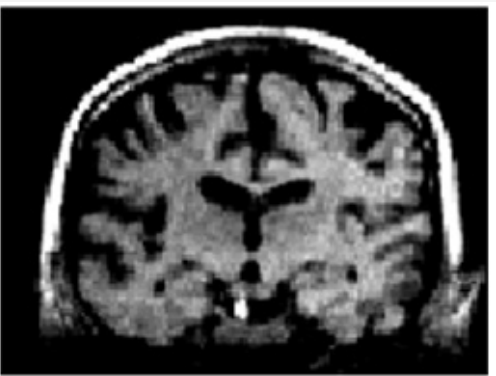

b. MRI image of the human brain of the Human Brain [12]. 
There are many observers from diverse fields of research with singular, holistic or complementary perspectives on the Internet as a global-mind and global spirit-phenomenon. The Internet is an integrated entity with pathways of development which are in a constant state of adaptation. The actively supported websites are kept alive by repeated "hits' or "visits" and those websites which receive limited visits cease to function or remain in a static state. A map of the World Wide Web has pattern similarities to a neuronal map of the brain (Figures 3\&4).



This is a graph of how the Internet might look as a packet of data spidered thru the bulk of the Internet infrastructure.

Dr. Ben Goertzel summarizes a theory presented in Neural Darwinism by biologist Gerald Edelman which theorizes brain mapping with the following hypothesis: The large-scale dynamics of the brain is dominated by the natural selection of maps. Those maps which are active when good results are obtained are strengthened, those maps which are active when bad results are obtained are weakened. And maps are continually mutated by the natural chaos of neural dynamics, thus providing new fodder for the selection process. By use of computer simulations we have shown that formal neural networks obeying this rule can carry routinely fairly complicated acts of perception [1].

In creating internet Intelligence mathematician Dr. Goertzel whose current focus is on the development of a web based artificial intelligence (AI) comments on the Internet versus brain: It's simple enough to see that the net, as a whole has the very same network structure that modern AI theorists, with their neural nets and semantic networks, have simulated within individual serial computers for the purpose of modeling brain and mind processes. The Internet's nodes are more complex than individual neurons, having more of the computational power of neuronal modules. And, the packets sent around the net are more complex than the jolts of charge sent around the brain - they are more similar, perhaps, to coherent nonlinear spatially distributed electromagnetic waves passing through the brain. But, in essence, they are quite similar systems: they're self-organizing networks, in which information is carried and computation is carried out both locally and in the global, distributed fashion.
And, continuing with his thought that the network is a computer is the mind: We see, then, that there is a harmony between the brain and the Internet on a fairly deep philosophical level. In both cases, we have a fixed underlying substrate consisting of nodes and links - neurons and synapses on the one hand, computers and cable on the other. In both cases, one has two kinds of dynamics constituting the process level of being - dynamics within the individual neuron or neuronal module, together with electrical and chemical flow between neurons; and computation within the individual computer, together with flow of sound and light along cables between computers [2].

\section{Intelligent systems: brain neurons and computer cards}

The following diagrams illustrate a striking resemblance in design. The firstillustration is a computer card which is programmed to serve a specific function in the computer communication system. The second illustration is a map plan drawing of the neurons in the brain which function as the olfactory communication pathway (Figure 5, 6).
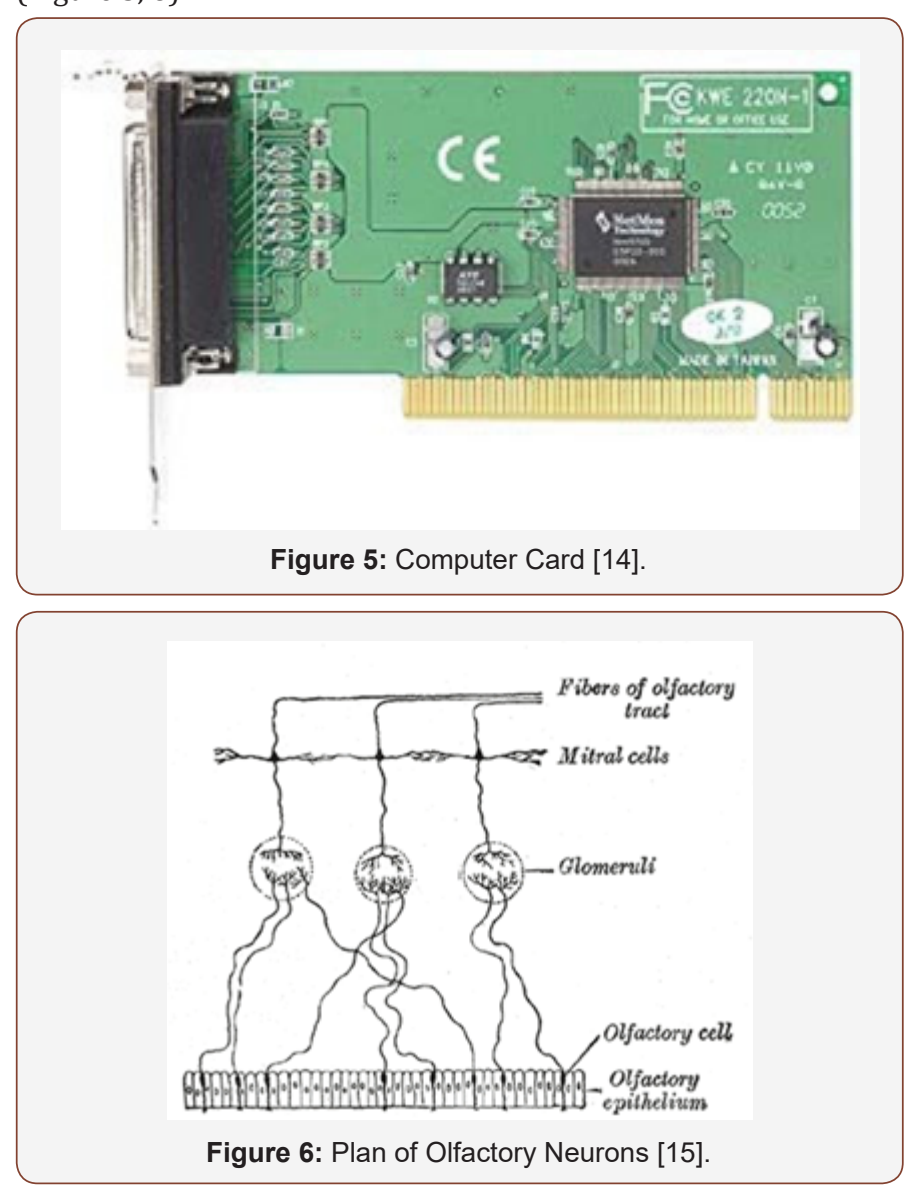

\section{Internet evolution: digital nervous system}

Is Internet email communication the micro-pathways which when accumulated into a whole organism emerge as a macroevolved entity? In Evolution without Selection, biologist Lima de Faria observes that the development of organisms is controlled by universal organizing processes. In Linked: The New Science of Networks physics professor, Albert-Laszlo Barbarsi using a simple mathematical transformation, substituted "fitness" for "energy" assigning an individual energy level to each World Wide Web 
node in the fitness model. The unexpected result was a precise mathematical correspondence between the networks of the web and the quantum mechanics world of Bose-Einstein Condensation. A simple definition of quantum mechanics is the study of light as little discrete packets of quanta or photons. A Bose-Einstein Condensation is an odd phenomenon in which the little discreet packets stop behaving as individuals and begin to flow together as if they are all one. A similar process occurs as a laser produces coherent light. These subatomic photon laws are encountered in the microscopic world of subatomic physics not in the macroscopic world of the Internet nodes and links which are macro objectsrouters, cables and hardware. This discovery illuminates the science of complex evolving networks as being analogous to the realm of physics.

In the Evolving Mind, Goertzel uses mathematical theory, “... to present some new ideas about the nature of the evolving systems." And, continues with his theory on biological patterns which sounds akin to the evolving pattern of the Internet. The important concept of emergent pattern is defined: a pattern emerges between two entities if it is present in the combination of the two entities, but not in either of the entities separately. And the structural complexity of an entity is defined as the "total amount" of pattern in it. If the Metapattern is accepted, then these two concepts become essential to any of analysis of biological reality. The new forms which are generated by structural instability must interact with one another in a structured, systematic way, to form a cohesive evolutionary system [3].

\section{World wide web as an ecosystem}

A working postulate is that the pattern of Internet based communication within the World Wide Web combines two "entities" which interact as a complex reality to form a structured cohesive system. This is analogous to the evolutionary pattern observed in many natural and social systems. Might this definition also be applied to the Internet as an emerging social intelligence?

The random inter-self-organization and the chaotic intra-selforganization of the Internet follows the "systems-theory" approach to evolution of systems presented by ecological scientist, Howard Odum. The operational nature of the self-structuring system of Internet communication aligned with this systems-theory leads to the assumption of an existence of an underlying working intelligence.

Dr. Goertzel states his definition of "self-structuring" systems: I conjecture that brains, minds, ecosystems and societies fall into this category. They are not chaotic, and yet they are not orderly in the classical sense either - they are systems of unpredictably fluctuating, self-organizing structure.

This definition forms a link between intelligence and system complexity: it suggests the hypothesis that intelligence is possible only in a self-structuring environment. And this meshes very nicely with the idea, implicit in my theory of mind-that intelligent systems are necessarily self-structuring [3].

\section{Is the medium the message?}

Cultural theorist Jean Gebser in his work on Integral Consciousness spoke of a fifth stage where intelligent network computer technology will catapult humanity into a new phase of consciousness; computer networks will be a higher state of consciousness and induce humans to move into a higher state of consciousness as well. Goertzel's thoughts are, "The leverage of globally distributed nonlinear interactions between human minds and digital minds, will in itself lead to forms of knowledge that are hitherto unprecedented." And, maybe those precedents already exist.

Parallel thought is emerging in research fields of spirituality, physics and computer science. Reading books from these multidisciplinary sciences one can easily transpose words to find that the hypothesis and conclusions sound the same. Theoretical physicist David Bohm's thoughts bridge science and spirit in his view that everything has a physical and mental aspect. He sees the fundamental activity of existence as a process of "projection and injection" in the creation of a form. Projection is the action of the mind to birth an unmanifest concept (implicate) into the manifest world (explicate). Injection is the feedback loop of the explicate reality mirroring back to the mental realm of the implicate for evaluation and refinement of objective/subjective coherence and resonance. The fundamental activity of the world is creating form through the dance of this circulating process. This passage on spirituality from Bridging Science and Spirit, if one transposes words, can be read as a descriptor of the operating order of the Internet.

In Bohm's view we are defining the structure or pattern of the universe, the shape and organization of a system, as distinguished from matter itself. Since influencing [matter] does not take place through the explicate order, it is nonlocal and takes place in the implicate order. Since the implicate order is not local, similar forms resonate and are connected regardless of their location in time and space. Through the process of resonance, particular forms are reinforced and appear in the manifest world as one stable form. The stable form can appear anywhere, and all similar forms influence each other in the implicate order (or the unmanifest) [4].

\section{The power-law of life}

I think computer viruses should count as life. I think it says something about human nature that the only form of life we have created so far is purely destructive. We've created life in our own image. $\sim$ Stephen Hawking

Life is defined by Webster's dictionary as, "-the condition which distinguishes organic from inorganic objects, being manifest by growth through reproduction and the power of adaptation to environment through changes originating internally." The Internet, like the living cell shares a common core process of growth, selfreproduction and adaptation-activities which take place internally. In Linked: The New Science of Networks, physics professor AlbertLaszlo Barabasi notes that, "While entirely of human design, the 
Internet now lives a life of its own. It has all the characteristics of a complex evolving system, making it more similar to a cell than a computer chip." [5].

Most complex networks in nature have power-law degree distribution-with many nodes and only a few links; or a few hubs with a large number of links. The degree distribution of a "random" network follows a bell-curve with most nodes having the same number of links.

Physicists Romualdo Pastor-Satorras and Alessandro Vespignani have identified computer networks as a class of "scalefree" networks in which there is no characteristic, average number of connections to the many nodes of the network. In a regular gridlike network, like a street map, each node is joined to the same number of neighboring nodes -- four, in a square grid. In a random network, where all the nodes are connected at random, there is a well-defined average number of connections among the nodes, and the number that have many more connections than average is insignificantly small.

Recent computer network research has come to the surprising discovery that the Web, like most complex networks of nature follows a power-law degree distribution of a

scale-free network-a description given to emergent ecosystems.

Power laws regularly greet us in critical phenomena and describe, for example, the freezing of water or the ordering of spins in a magnet. But there is a crucial difference between these systems and evolving networks. In critical phenomena the exponents are fixed and universal, i.e. they cannot be tuned easily by modifying some parameters in the system. In networks, however, the exponent G can be changed continuously by changing almost every parameter that governs the link and nodes. Thus, universality as we know it is absent. However, most complex systems share the same dynamical character as evolving networks, indicating that their topology and evolution cannot be divorced one from the other.

(Figure 7).

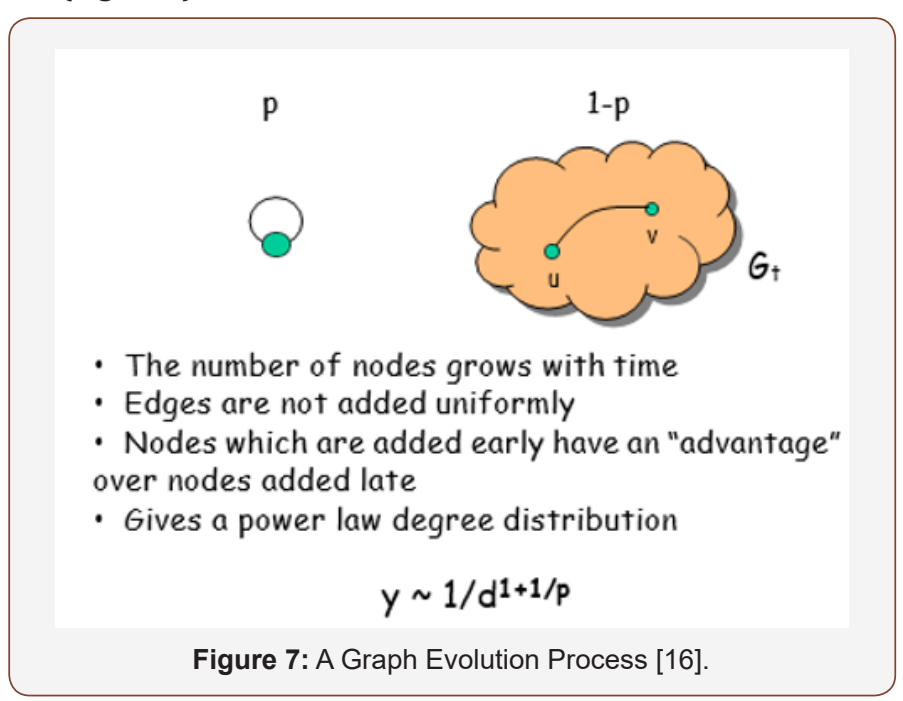

\section{The web of life}

Interdependent systems of life are linked by some form of communication. Nature strives to achieve robustness through inter-connectivity. A hierarchy of hubs and nodes keeps the Internet connected, followed by lesser connected hubs and links, forming the life of the World Wide Web. "No central node sits in the middle of the web. There is no single node whose removal could break the web." [5].

Many scientists are predicting that the robustness of the web network gives it a unique ability to survive under very high error rates. Physics professor, Marc Buchanan comments in Nexus on the inter-connectivity of nodes and links

...The Internet actually harbors a hidden order. The Internet is not the only network underlying the Information Revolution. It is an entire physical entity-sprawling network of computers linked together by transmission lines. The Internet is more or less pure hardware. By contrast, the World Wide Web is rather more ethereal... you can click onto a Web site and be transported elsewhere. If you will, the World Wide Web is the face of the Internet [6].

\section{The dream of life}

Dialogue from the 1968 and 1984 films: 2001: A Space Odyssey and 2010: The Year We Make Contact.

HAL: “Good afternoon, gentlemen. I am a HAL 9000 computer. I became operational at the

H.A.L. lab in Urbana, Illinois on the 12th of January 1992."

HAL (answering questions): "Let me put it this way, Mr. Amer the 9000 series is the most reliable computer ever made. No 9000 computer has never made a mistake or distorted information. We are all, by any practical definition of the words, foolproof and incapable of error."

HAL (to Dr. Chandra): "I know that you were planning to disconnect me, and I'm afraid that's something I cannot allow to happen."

HAL (eventually agreeing to be disconnected): "Dr. Chandra?" Dr. Chandra: "Yes HAL?"

HAL: "Will I dream?"

\section{Feminine intuition and the right brained internet}

Surgeon and author, Leonard Shlain. has a collectiveunconscious observation, "The world of cyberspace is a computergenerated extension of the human mind into another dimension." This is a humanistic linkage statement for the inclusion of intuition and spirituality into the emerging web-life of the Internet. Shlain writes in his book, The Alphabet Versus the Goddess that, "The computer's processes have unwittingly advanced the cause of women..."

The computer and the Internet will once again reconfigure the brains of those that use them. Typing is a two-handed activity that requires input from both sides of the brain. Writing requires only the dominant hand. The use of the mouse by the right hand necessitates the activation of the right-hemispheric and visualspatial skills. The World Wide Web and the Internet are not linear, they are holistic. 
All ancient deities associated with webs and nets were goddesses. Many of the processes we use to operate the computer are inherently feminine [7-11].

\section{Internet degrees of separation}

Recent network theory according to Albert Laszlo-Barabasi states that the Internet distance (clicks or hops) between people is nineteen-degrees of separation. In November 2002, I asked a colleague who lives an approximate 30 miles distance from me to send an email to me and then trace the visual route and hops between his personal computer and my personal computer. The social Internet distance between my colleague and I was traced as twelve-degrees (12 hops) of Internet separation [17,18].

\section{Discussion}

The statistical results of this specific study are significant; however, it is inconclusive that the results are directly associative to the Internet as a transmitter/holder of energy. Other considerations and possibilities include:

1. The email might have served as a "witness" - an object in the material and energetic worlds that serves to connect the sender and receiver through psychic links. That is, the receivers may have connected directly with the sender, using the email as something akin to a homing beacon. However, the time of emails sent and received was random; and the receivers did not know who the "sender" was (in the information obtained from the final questionnaires many responded that they assumed it was the author who was the sender). Of more important note is that within the research protocol the professional intuitive was not designed as the "sender" - the Internet was the designated sender/carrier of the encapsulated healing energy. The professional intuitive reported that he did not focus on any receivers, as his job was to simply encapsulate the CD- ROMS with healing energy, and then place each CD-ROM into the computer and push the send button.

2. This study may reflect the experimenter's expectations. The possibility that the experimental results were influenced by precognitive effects is a consideration that might follow the premise of Decision Augmentation Theory.

3. The test subjects were accessing a constant energy archival medium- working with a postulate that time is nonlinear and nonlocal the subjects may have the skill to access information outside of linear time. The Internet may participate in this phenomenon in ways that might be explained within the Zero Point Field, Akashic Records or the Collective Unconscious theories.

4. Addressing a replication consideration: This project states that the intention was to use finely tuned human measurement instruments-the intuitives trained in the same protocols as the sender. The study has confirmed the hypothesis to a limited degree and only for the Intuition Medicine trained intuitive subjects. However, this would not limit the replication of the research, as another group of energy practitioner, who were, for example, trained in Reiki or Johrei, could also follow the same protocols.

\section{Is the Internet Conscious?}

Personal Computer life is a constant interaction with the wire and cable-based Internet entity, even given that, it is difficult for many web users to engage with their computer system as a lifeless form. Questions to consider: Given that the Internet has all the characteristics of a complex evolving system does this make the Internet more similar to a human cell rather than a computer chip? Is Internet email communication the micro-pathways which when accumulated into a whole organism operate as a macro-evolved entity? Who is the caster of this web net?

Are those who are gathered into this web net conscious of the implications of a global paradigm shift in human evolution? What is occurring on an individual basis within this web net, and what is being born as a whole entity?

We are living and intentionally creating extraordinary events in human evolution. Internet research contributes directly to the writing of our history as a species and discovers new language to articulate possibilities of science, technology, and the human potential.

\section{Acknowledgement}

None.

\section{Conflicts of Interest}

No conflict of interest.

\section{References}

1. Gebser J (1986) The ever-present origin: Part one, foundation of the aperspectival world, Part two, manifestations the aperspectival world (English Series no .1). Athens OH: Ohio University Press, USA.

2. Goertzel B (2002) Creating Internet intelligence. Plenum Publishing, New York, USA.

3. Goertzel B (1993) The evolving mind: The world futures general evolution studies (Vol 6). PA: Gordon Breach Science Publishers, Langhorne, USA.

4. Friedman N (1990) Bridging science and spirit: Common elements in David Bohm's physics, the perennial philosophy and Seth. St Luis MO: Living Lakes Books.

5. Barabási AL (2002) Linked: The new science of networks. Perseus Publishing: Massachusetts, Cambridge, UK.

6. Buchanan M (2002) Nexus: Small worlds and the groundbreaking size of networks. WW Norton: New York, USA.

7. Shlain L (1998) The alphabet versus the Goddess: The conflict between word and image. Penguin Putnam, New York, USA.

8. Bohm D (1996) Wholeness and the implicate order. Routledge, New York, USA.

9. Edelman GM (1988) Neural Darwinism: The theory of neuronal group selection. Basic Books: New York, USA.

10. Jung CG (1981) The archetypes and the collective unconscious by $M$ Fordham (Eds.) \& RFC Hull (Eds.) NJ: Princeton University Press, Princeton, New York, USA, 9(1).

11. Odum HT (1983) Systems Ecology. Wiley: New York, USA. 
12. (2003) MRI image of the human brain.

13. (2003) Digital image of the Internet.

14. (2003) Computer card.

15. (2003) Plan of olfactory neurons.
16. (2001) The physics of the Web.

17. (2003) Academy of Intuition Medicine ${ }^{\circledR}$.

18. (2003) The Physics of the Web. 